\title{
A Cross-Sectional Study Of The Association Between Skin Tags And Vascular Risk Factors In Patients With Severe And Complicated Obesity
}

\section{Clarissa Ern Hui Fang}

Galway University Hospitals

\section{Catherine Crowe}

Galway University Hospitals

Annette Murphy

Galway University Hospitals

\section{Martin O’Donnell}

National University of Ireland Galway

Francis Martin Finucane ( $\nabla$ francis.finucane@hse.ie)

Galway University Hospitals

\section{Research note}

Keywords: Skin tags, Acrochordons, Severe obesity, Bariatric, Hypertension, Type 2 Diabetes

Posted Date: January 8th, 2020

DOI: https://doi.org/10.21203/rs.2.20372/v1

License: (c) (i) This work is licensed under a Creative Commons Attribution 4.0 International License.

Read Full License

Version of Record: A version of this preprint was published at BMC Research Notes on March 16th, 2020.

See the published version at https://doi.org/10.1186/s13104-020-05006-4. 


\section{Abstract}

Objective: We sought to determine whether the presence of cervical or axillary skin tags was associated with an adverse cardiovascular risk profile in Irish adults with severe obesity.

Results: We conducted a cross-sectional study of patients attending our regional bariatric centre. Of 167 patients, 100 (31\% male, 37\% with type 2 diabetes, $36 \%$ on lipid lowering therapy, $41 \%$ on antihypertensive therapy) agreed to participate. 85 patients had cervical or axillary tags while 15 had none. Those with any skin tags had higher systolic blood pressure $(138.0 \pm 16.0$ versus $125.1 \pm 8.3 \mathrm{mmHg}$, $\mathrm{p}=0.003)$ and higher glycated haemoglobin $(\mathrm{HbA1c})(46.5 \pm 13.2$ versus $36.8 \pm 3.5 \mathrm{mmol} / \mathrm{mol}$, $\mathrm{p}=0.017) .94 .6 \%$ of patients with diabetes, compared to $79.4 \%$ of those without diabetes had skin tags $(p=0.039) .45 .8 \%$ of patients with skin tags compared to $13.3 \%$ with no tags were on antihypertensive therapy $(p=0.018)$. Skin tags were not associated with any differences in lipid profiles. In Irish adults with severe obesity, skin tags are associated with higher systolic blood pressure and $\mathrm{HbA} 1 \mathrm{c}$ and a higher prevalence of diabetes and hypertension, consistent with increased vascular risk, though differences in lipid profiles were not found.

\section{Introduction}

Obesity is known to cause increased mortality(1) and morbidity(2) from diseases such as type 2 diabetes. However, there is heterogeneity in the relationship between excess body fat and disease risk. For example, some patients develop diabetes at a relatively low level of adiposity while others never develop diabetes, even with severe obesity.(3) Insulin resistance, the key defect linking obesity and type 2 diabetes,(4) also drives increased vascular risk.(5) However precise measurement of insulin resistance in routine clinical practice is usually not feasible.(6) Cutaneous markers of insulin resistance include acanthosis nigricans, androgenetic alopecia, acne, hirsutism and skin tags (acrochordons).(6-8) These benign, pedunculated papular lesions are commonly located on the neck, axillae, eyelids and groin. While their association with diabetes has long been recognized,(9) skin tags are not usually considered in the clinical assessment of patients or emphasized in clinical practice guidelines and, rather like insulin resistance itself, they represent an abstract academic consideration at the patient's bedside rather than a relevant and quantifiable indicator of vascular risk. Nonetheless, in our clinical practice we have found skin tags to be a useful marker of insulin resistance in patients presenting with atypical diabetes phenotypes. $(10,11)$

Skin tags are thought to arise from high levels of insulin and associated growth factors causing proliferation of epidermal fibroblasts. They have been associated with various indicators of increased vascular risk including an adverse lipid profile,(12-16) insulin resistance, $(17,18)$ impaired glucose metabolism, $(19,20)$ elevated blood pressure $(14,21)$ and components of the metabolic syndrome. $(7,14$, $21,22)$ However to date most studies have been done in non-white ethnic groups or those without severe obesity. Specifically, the clinical significance of the presence of skin tags in White European patients with severe obesity has not been determined previously. Also, the extent to which the presence of skin tags 
predicts an adverse cardiovascular risk profile in this group is not clear. We sought to determine whether skin tags were associated with an adverse vascular risk profile in a predominantly white population of Irish adults attending our bariatric centre for management of severe obesity.

\section{Materials And Methods}

This was a single centre cross-sectional study. The study population included a convenience sample of patients $\geq 18$ years old with a BMl (body mass index) $\geq 40 \mathrm{~kg} \mathrm{~m}^{-2}$ (or $\geq 35$ with co-morbidity) who were attending a hospital-based bariatric outpatient department. The study was approved by the local research ethics committee. The numbers of patients approached and recruited have been reported in line with STROBE guidelines.(23) At assessment, fully informed written consent was obtained from each participant. The use of cardioprotective medications such as antihypertensive and lipid lowering drugs was noted. Weight was measured using a Seca ${ }^{\circledR}$ scale and height with a Seca ${ }^{\circledR}$ Leicester stadiometer. Systolic blood pressure (SBP) was measured with an Omron ${ }^{\circledR} 705 I T$ oscillometric device in both arms after the patient was sitting comfortably for five minutes. Ethnicity was self-reported.

\section{Laboratory analysis}

All blood samples were analysed locally in the Galway University Hospitals' Department of Clinical Biochemistry (certified to ISO 151892007 accreditation standard). HbA1c was measured with high performance liquid chromatography(Menarini® HA8160 auto-analyzer). Total cholesterol was measured using the CHOP-PAP method. High density lipoprotein (HDL)-cholesterol and triglycerides were measured using the enzymatic and the GPO-PAP methods, respectively (Roche COBAS $® 8000$ modular analyzer). Low density lipoprotein (LDL)-Cholesterol was derived with the Friedewald equation.(24) We derived the triglyceride:HDL-cholesterol ratio (THDLR) as a surrogate marker of insulin resistance.(25)

\section{Dermatological assessment}

All patient dermatological assessments were conducted by a single investigator (CC) having undergone a period of clinical instruction in acrochordon assessment by a consultant dermatologist (AM) and according to a standard operating procedure. Each participant was given the opportunity to have a chaperone present for the examination. Where acrochordons were present, their location (axillary or cervical), number and size were documented.

Statistical analysis

Differences between participants with versus those without skin tags were determined using the unpaired t-test and differences in proportions were assessed with the Chi-Square test. Where we found differences in those with versus without skin tags, the influence of confounders such as medication usage, sex, age and $\mathrm{BMI}$ were assessed using binomial logistic regression with the presence of any skin tags being the dependent variable. We used linear- as well as ordinal- regression to determine whether the number of skin tags (either as a continuous or categorical dependent variable) was associated with vascular risk 
factors. A p-value of $<0.05$ was deemed to be statistically significant. All analyses were performed using SPSS version 24.

\section{Results}

Between December 2012 and June 2014, 164 patients attended our facility and of these, 100 agreed to participate in the study. The male: female ratio was 31:69. There was one patient of Black African descent, five of Irish Traveler descent and 94 of White European descent. The mean age was $53.7 \pm 11.3$ (range 31.1-80) years. The mean number of skin tags was $10.5 \pm 18.4$ (range $0-135$ ). Tags were present in 85 patients and absent in 15 . Skin tags were present only in the axillae in 30 patients, only on the neck in 11 patients and 44 patients had skin tags in both the axillae and the neck. 62 patients had three or more skin tags while 38 had two or fewer. 12 patients were current smokers while 88 were previous or never smokers. 14 patients reported a current or past history of sleep apnea while 4 had previous bariatric surgery. 41 patients were currently taking antihypertensive therapy (59 were not), 37 were taking medication for type 2 diabetes (none had type 1 diabetes) and 36 patients were taking lipid lowering therapy (either statin or fibrate). Of the 37 patients with diabetes, 35 had skin tags ( $94.6 \%)$, compared to 50 of 63 patients without diabetes $(79.4 \%, p=0.039)$. Put another way, $59 \%$ of those with skin tags, compared to $13 \%$ of those with no tags had diabetes. Skin tags were present in $79.7 \%$ of female and $96.8 \%$ of male patients $(p=0.027) .45 .8 \%$ of patients with skin tags compared to $13.3 \%$ with no tags were on antihypertensive therapy $(p=0.018)$, while $38.8 \%$ and $20 \%$, respectively, were on lipid lowering therapy $(p=0.16)$.

The anthropometric and metabolic characteristics of study participants with any skin tags compared to those with no skin tags are presented in Table 1. Those with any skin tags had higher SBP and fasting blood glucose and a higher HbA1c. There was a (statistically non-significant) trend to higher triglyceride:HDL cholesterol ratio in those with skin tags, consistent with them being more insulin resistant. Likewise, there were non-significant trends to increased weight and diastolic blood pressure in those with versus those without any skin tags. 
Table 1

Anthropometric and metabolic characteristics of adults with severe obesity comparing those with either axillary or cervical skin tags to those with no skin tags.

\begin{tabular}{|llllll|}
\hline Variable & \multicolumn{2}{l}{ No Skin tags } & \multicolumn{2}{l}{ Any Skin tags } & P-value \\
\hline & $\mathrm{n}$ & 15 & $\mathrm{n}$ & 85 & \\
\hline Systolic blood pressure (mmHg) & 125.1 & \pm 8.3 & 138.0 & \pm 16.0 & $<0.001$ \\
\hline Diastolic blood pressure (mmHg) & 80.4 & \pm 9.7 & 85.6 & \pm 9.7 & 0.094 \\
\hline Total Cholesterol (mmol/L) & 5 & \pm 1.0 & 4.6 & \pm 1.2 & 0.19 \\
\hline LDL Cholesterol (mmol/L) & 3.1 & \pm 0.9 & 2.6 & \pm 1.2 & 0.136 \\
\hline HDL Cholesterol (mmol/L) & 1.3 & \pm 0.3 & 1.2 & \pm 0.3 & 0.329 \\
\hline Triglycerides (mmol/L) & 1.5 & \pm 0.7 & 1.8 & \pm 1.1 & 0.351 \\
\hline Triglyceride:HDL & 1.3 & \pm 0.6 & 1.7 & \pm 1.4 & 0.054 \\
\hline Total Cholesterol (mmol/L)+ & 5.3 & \pm 0.8 & 4.8 & \pm 1.2 & 0.155 \\
\hline LDL Cholesterol (mmol/L) + & 3.4 & \pm 0.7 & 2.8 & \pm 1.1 & 0.103 \\
\hline HDL Cholesterol (mmol/L) + & 1.2 & \pm 0.2 & 1.2 & \pm 0.3 & 0.993 \\
\hline Triglycerides (mmol/L) + & 1.6 & \pm 0.7 & 1.7 & \pm 1.0 & 0.712 \\
\hline Triglyceride:HDL+ & 1.3 & \pm 0.6 & 1.6 & \pm 1.4 & 0.543 \\
\hline Total Cholesterol (mmol/L)++ & 3.9 & \pm 0.8 & 4.2 & \pm 1.3 & 0.666 \\
\hline LDL Cholesterol (mmol/L)++ & 1.9 & \pm 0.3 & 2.3 & \pm 1.2 & 0.587 \\
\hline HDL Cholesterol (mmol/L)++ & 1.5 & \pm 0.7 & 1.1 & \pm 0.3 & 0.128 \\
\hline Triglycerides (mmol/L)++ & 1.1 & \pm 0.3 & 1.9 & \pm 1.2 & 0.288 \\
\hline Triglyceride: HDL ratio++ & 0.9 & \pm 0.5 & 1.8 & \pm 1.5 & 0.306 \\
\hline Glucose (mmol) & 5.1 & \pm 0.5 & 6.6 & \pm 2.5 & $<0.001$ \\
\hline HbA1c (mmol/mol) & 37.8 & \pm 3.5 & 46.5 & \pm 13.2 & $<0.001$ \\
\hline & & & & & \\
\hline
\end{tabular}

Data are presented as means \pm standard deviations.

*Denotes subgroup without diabetes (No Skin tags $n=13$, Skin tags $n=50$ ).

**Denotes subgroup with diabetes (No Skin tags $n=2$, Skin tags $n=35$ ).

${ }^{+}$Denotes subgroup not on lipid lowering agent (No Skin tags $n=12$, Skin tags $n=52$ ).

${ }^{++}$Denotes subgroup on lipid lowering agent (No Skin tags $n=3$, Skin tags $n=33$ ). 


\begin{tabular}{|c|c|c|c|c|c|}
\hline \multirow{2}{*}{$\begin{array}{l}\text { Variable } \\
\text { Glucose (mmol)* }\end{array}$} & \multicolumn{2}{|c|}{ No Skin tags } & \multicolumn{2}{|c|}{ Any Skin tags } & \multirow{2}{*}{$\begin{array}{l}\text { P-value } \\
0.189\end{array}$} \\
\hline & 5 & \pm 0.5 & 5.7 & \pm 1.9 & \\
\hline $\mathrm{HbA} 1 \mathrm{c}(\mathrm{mmol} / \mathrm{mol}) *$ & 36.8 & \pm 2.2 & 40.7 & \pm 10.5 & 0.201 \\
\hline Glucose $(\mathrm{mmol}) \star \star$ & 5.6 & \pm 0.4 & 7.9 & \pm 2.7 & 0.244 \\
\hline $\mathrm{HbA} 1 \mathrm{c}(\mathrm{mmol} / \mathrm{mol})^{\star \star}$ & 44 & \pm 4.2 & 53.9 & \pm 12.7 & 0.289 \\
\hline Weight $(\mathrm{kg})$ & 122.1 & \pm 15.3 & 130.2 & \pm 27.4 & 0.113 \\
\hline Height $(\mathrm{m})$ & 1.61 & \pm 0.07 & 1.68 & \pm 0.11 & 0.007 \\
\hline Waist circumference $(\mathrm{cm})$ & 133.7 & \pm 12.8 & 136.3 & \pm 18.9 & 0.611 \\
\hline BMI (kg/m2) & 47.1 & \pm 6.6 & 46 & \pm 7.8 & 0.61 \\
\hline Functional Capacity (MET max) & 6 & \pm 1.7 & 6.1 & \pm 2.0 & 0.859 \\
\hline \multicolumn{6}{|c|}{ Data are presented as means \pm standard deviations. } \\
\hline \multicolumn{6}{|c|}{ *Denotes subgroup without diabetes (No Skin tags $n=13$, Skin tags $n=50$ ). } \\
\hline \multicolumn{6}{|c|}{$\star \star$ Denotes subgroup with diabetes (No Skin tags $n=2$, Skin tags $n=35$ ). } \\
\hline \multicolumn{6}{|c|}{${ }^{+}$Denotes subgroup not on lipid lowering agent (No Skin tags $n=12$, Skin tags $n=52$ ) } \\
\hline
\end{tabular}

Results from logistic regression analyses, with the presence or absence of any skin tags as the binary dependent variable are shown in Table 2. For every rise of $1 \mathrm{mmHg}$ in SBP, the likelihood of having any skin tags increased by $7.5 \%(p=0.005)$ while patients with hypertension were 5.5 times more likely to have skin tags than patients with normal SBP $(p=0.031)$. However, the association with hypertension was no longer significant after adjusting for age and sex, both of which had a borderline significant association with skin tag presence, as shown in Table 2. There were non-significant trends to men being 7.6 times more likely than women to have skin tags $(p=0.055)$ while for every year older, there was a nonsignificant trend to a $5.1 \%$ increase in the likelihood of skin tags $(p=0.067)$. Similarly, there was a nonsignificant trend to a 4.55 -fold increased likelihood of any skin tags in patients with diabetes compared to those without diabetes $(p=0.055)$. Those with tags had a higher HbA1c, but not after adjusting for age and sex. The borderline difference observed in the THDLR using the unpaired t-test was not replicated using logistic regression in unadjusted or adjusted analyses. 
Table 2

Logistic regression model comparing the influence of various clinical features on the likelihood of having any skin tags versus none, in patients with severe obesity.

\begin{tabular}{|c|c|c|c|c|c|c|}
\hline Variable & Beta (logit) & S.E. & Exp (Beta) & 95\% C.I. & & p-value \\
\hline Sex & 2.033 & 1.060 & 7.636 & {$[0.957$} & 60.939] & 0.055 \\
\hline Age & 0.05 & 0.027 & 1.051 & {$[0.997$} & 1.109] & 0.067 \\
\hline Diabetes status & 1.515 & 0.791 & 4.55 & {$[0.966$} & 21.439] & 0.055 \\
\hline Diabetes status* & 1.357 & 0.808 & 3.883 & {$[0.797$} & 18.906] & 0.093 \\
\hline Hypertension status & 1.707 & 0.790 & 5.511 & {$[1.171$} & 25.929] & 0.031 \\
\hline Hypertension status* & 1.316 & 0.822 & 3.728 & {$[0.745$} & 18.666] & 0.109 \\
\hline SBP & 0.073 & 0.026 & 1.075 & {$[1.022$,} & 1.132] & 0.005 \\
\hline SBP* & 0.064 & 0.026 & 1.066 & {$[1.013$,} & $1.122]$ & 0.014 \\
\hline $\mathrm{HbA1c}$ & 0.107 & 0.05 & 1.113 & {$[1.01$} & 1.227] & 0.031 \\
\hline $\mathrm{HbA} 1 \mathrm{c}^{*}$ & 0.082 & 0.048 & 1.086 & {$[0.988$} & 1.194] & 0.089 \\
\hline THDLR & 0.376 & 0.338 & 1.457 & {$[0.752$} & $2.824]$ & 0.265 \\
\hline THDLR* & 0.271 & 0.313 & 1.311 & {$[0.71$} & 2.418 & 0.387 \\
\hline \multicolumn{7}{|c|}{ * Adjusted for age and sex. } \\
\hline \multicolumn{7}{|c|}{$\begin{array}{l}\text { Beta is the logit or estimated log odds of having any skin tags for every one unit rise in the variable } \\
\text { measure. }\end{array}$} \\
\hline \multicolumn{7}{|c|}{ S.E: Standard error for Beta. } \\
\hline \multicolumn{7}{|c|}{$\begin{array}{l}\text { Exp (B) is the exponential of Beta. (So for example for every } \mathrm{mmHg} \text { rise in systolic blood pressure, } \\
\text { there is a } 7.5 \% \text { increased likelihood of having skin tags.) }\end{array}$} \\
\hline \multicolumn{7}{|l|}{ C.I: Confidence Interval } \\
\hline \multicolumn{7}{|c|}{ SBP: Systolic Blood Pressure } \\
\hline \multicolumn{7}{|c|}{ THDLR: Triglyceride: HDL-cholesterol ratio. } \\
\hline
\end{tabular}

In linear regression models with the number of skin tags being the dependent or outcome variable, male sex was strongly associated with skin tags $(B=15.85$ [8.61, 23.09], $p<0.001)$. In other words, men had on average 16 more skin tags than women. There was a borderline statistically significant association between age and skin tags $(B=0.32[-0.003,0.63], p=0.052)$ such for every ten years older, there were three more skin tags. Similar results to the findings from binary logistic and linear regression analyses were obtained when the number of skin tags was arbitrarily categorized and ordinal regression analyses were conducted (data not shown). 
Next, we compared anthropometric and metabolic characteristics in participants with both cervical and axillary skin tags to those who had no skin tags (Supplementary table 1). As before, there were significant differences in glucose and HbA1c, but in addition to SBP, diastolic blood pressure was also elevated and there was a significant difference in weight of $14.8 \mathrm{~kg}$ in those with axillary and cervical tags compared to those with none. We compared patients who had axillary skin tags versus those without axillary skin tags, with the only significant difference being greater height and weight in those with axillary skin tags (Supplementary table 2). Finally, we found that compared to patients without cervical skin tags, those with cervical skin tags had higher systolic and diastolic blood pressure, with higher BMI and a (borderline significant) higher waist circumference and weight (Supplementary table 3).

\section{Discussion}

We have shown that in a predominantly White European cohort of Irish adults with severe obesity, skin tags were more prevalent in patients with diabetes and hypertension and were associated with higher SBP and HbA1c. However, there were no differences in lipid profiles and the trend to an increased triglyceride:HDL cholesterol ratio in those with skin tags, suggestive of insulin resistance, did not reach statistical significance.

The population is predominantly White European, so while it limits the generalizability of the results to other ethnic groups, it also reduces random error from variations in hereditary influences on the relationship between skin tags and vascular risk factors. Given that the dermatological examination was done by a specially trained single operator (CC) according to a standard protocol and under expert dermatological guidance (AM), inter-observer subjectivity in acrochordon assessment and information bias have been minimized.

Our findings of elevated blood glucose and $\mathrm{HbA} 1 \mathrm{c}$ in patients with skin tags are consistent with observations in Indian $(19,21)$ and Turkish(20) cohorts. Similarly, impaired insulin sensitivity in those with skin tags has been described in Iranian(18) and Egyptian(17) cohorts. However the differences in the various components of the lipid profile were not more apparent in our study. Three different Indian studies $(12,13,16)$ and one from Turkey $(14)$ have found atherogenic lipid profiles associated with skin tags.

Our findings need to be retested in larger studies. The utility of skin tags as a meaningful sign of insulin resistance and increased vascular risk remains to be determined. Indeed, we have not sought to quantify directly insulin resistance in this study, though this would clearly have been valuable. Currently, insulin resistance is an abstract and poorly defined state in routine clinical practice, relying to some extent on the physician's "gestalt". Hence there is a need to identify and validate novel surrogate clinical and metabolic measures of insulin sensitivity and vascular risk, such as has already been done with waist circumference(26) and adipokines(27), respectively.

In summary our findings are novel in the context of skin tags being associated with an adverse cardiovascular risk profile in Irish adults with severe obesity. Further studies comparing differences in 
insulin sensitivity and cardiovascular risk in patients with skin tags seem warranted.

\section{Limitations}

The sample size was determined by a convenience sample of patients attending our bariatric service over 18 months, and we cannot assume that our results are generalizable to adults with severe obesity not attending hospital bariatric services - there may have been some selection bias in the population studied. We did not collect data on diabetes medications and these may have influenced THDLR. A larger number of patients would be helpful in more precisely elucidating the influence of sex, age and ethnicity on the relationship between tags and adverse phenotype.

\section{Abbreviations}

$\mathrm{HbA1c}$

glycated haemoglobin

$\mathrm{BMI}$

body mass index

SBP

systolic blood pressure

HDL

High density lipoprotein

LDL

Low density lipoprotein

THDLR

triglyceride:HDL-cholesterol ratio

\section{Declarations}

\section{Ethics approval and consent to participate}

An ethical application was submitted to the Galway University Hospital Ethics board on the 05/07/2013. The ethics committee approved the study on the 18/07/2013. The site at which this study was conducted on was covered by the clinical indemnity scheme.

\section{Consent for publication}

Not applicable

\section{Availability of data and materials}


Data analysed in this study are included in this published article and its supplementary information files.

\section{Competing interests}

In the past (up to 2017), Francis Finucane received honoraria, travel grants and has served on advisory boards for Novo Nordisk, Eli Lilly, Pfizer Inc., Sanofi-Aventis, Astra Zeneca, Merck-Serono, Boehringer Ingelheim, Janssen and Novartis. Catherine Crowe, Clarissa Fang, Annette Murphy and Martin O'Donnell have no competing interest to declare.

\section{Funding}

This research was supported by a Clinical Research Career Development Award from Saolta University Health Care Group to Francis Finucane as well as a project grant from Healthy Ireland, a Government of Ireland initiative.

\section{Author's contributions}

CF led the writing of the manuscript and statistical analysis. CC assisted with writing and revision of the manuscript and with statistical analysis and conducted the recruitment and clinical assessment of study participants. AM assisted with study design and revision of the manuscript. MOD assisted with the study design, the statistical analysis and with writing the manuscript. FF supervised writing and revision of the manuscript and statistical analysis and is the study guarantor. All authors approved the final version of the manuscript.

\section{Acknowledgements}

We are extremely grateful to all of the patients who participated in this study. We are also grateful to the staff at the Centre for Diabetes, Endocrinology and Metabolism at Galway University Hospitals, the staff at the HRB Clinical Research Facility, Galway and also the staff at Croi, the West of Ireland Cardiac Foundation, for their help with conducting this study.

\section{References}

1. Abramowitz MK, Hall CB, Amodu A, Sharma D, Androga L, Hawkins M. Muscle mass, BMI, and mortality among adults in the United States: A population-based cohort study. PLoS One. 2018;13(4):e0194697. 
2. Laaksonen MA, Knekt P, Rissanen $\mathrm{H}$, Harkanen $\mathrm{T}$, Virtala $\mathrm{E}$, Marniemi J, et al. The relative importance of modifiable potential risk factors of type 2 diabetes: a meta-analysis of two cohorts. Eur $\mathrm{J}$ Epidemiol. 2010;25(2):115-24.

3. Stefan N, Haring HU, Hu FB, Schulze MB. Metabolically healthy obesity: epidemiology, mechanisms, and clinical implications. Lancet Diabetes Endocrinol. 2013;1(2):152-62.

4. Shulman GI. Ectopic fat in insulin resistance, dyslipidemia, and cardiometabolic disease. The New England journal of medicine. 2014;371(23):2237-8.

5. Yip J, Facchini FS, Reaven GM. Resistance to insulin-mediated glucose disposal as a predictor of cardiovascular disease. J Clin Endocrinol Metab. 1998;83(8):2773-6.

6. Gonzalez-Saldivar G, Rodriguez-Gutierrez R, Ocampo-Candiani J, Gonzalez-Gonzalez JG, GomezFlores M. Skin Manifestations of Insulin Resistance: From a Biochemical Stance to a Clinical Diagnosis and Management. Dermatol Ther (Heidelb). 2017;7(1):37-51.

7. Karadag AS, Ozlu E, Lavery MJ. Cutaneous manifestations of diabetes mellitus and the metabolic syndrome. Clinics in dermatology. 2018;36(1):89-93.

8. Boza JC, Trindade EN, Peruzzo J, Sachett L, Rech L, Cestari TF. Skin manifestations of obesity: a comparative study. Journal of the European Academy of Dermatology and Venereology : JEADV. 2012;26(10):1220-3.

9. Kahana M, Grossman E, Feinstein A, Ronnen M, Cohen M, Millet MS. Skin tags: a cutaneous marker for diabetes mellitus. Acta dermato-venereologica. 1987;67(2):175-7.

10. Hui Fang CE, Rafey MF, Cunningham A, Dinneen SF, Finucane FM. Risperidone-induced type 2 diabetes presenting with diabetic ketoacidosis. Endocrinol Diabetes Metab Case Rep. 2018;2018.

11. Small C, Egan AM, Elhadi EM, O'Reilly MW, Cunningham A, Finucane FM. Diabetic ketoacidosis: a challenging diabetes phenotype. Endocrinol Diabetes Metab Case Rep. 2017;2017.

12. Wali V, Wali VV. Assessment of Various Biochemical Parameters and BMI in Patients with Skin Tags. J Clin Diagn Res. 2016;10(1):BC09-11.

13. Idris S, Sunitha S. Assessment of BMI, Serum Leptin Levels and Lipid Profile in Patients with Skin Tags. J Clin Diagn Res. 2014;8(9):CC01-3.

14. Akpinar F, Dervis E. Association between acrochordons and the components of metabolic syndrome. European journal of dermatology : EJD. 2012;22(1):106-10.

15. Senel E, Salmanoglu M, Solmazgul E, Bercik Inal B. Acrochordons as a cutaneous sign of impaired carbohydrate metabolism, hyperlipidemia, liver enzyme abnormalities and hypertension: a casecontrol study. Journal of the European Academy of Dermatology and Venereology : JEADV. 2011.

16. Gorpelioglu C, Erdal E, Ardicoglu Y, Adam B, Sarifakioglu E. Serum leptin, atherogenic lipids and glucose levels in patients with skin tags. Indian journal of dermatology. 2009;54(1):20-2.

17. Shaheen MA, Abdel Fattah NS, Sayed YA, Saad AA. Assessment of serum leptin, insulin resistance and metabolic syndrome in patients with skin tags. Journal of the European Academy of Dermatology and Venereology : JEADV. 2012;26(12):1552-7. 
18. Jowkar F, Fallahi A, Namazi MR. Is there any relation between serum insulin and insulin-like growth factor-I in non-diabetic patients with skin tag? Journal of the European Academy of Dermatology and Venereology : JEADV. 2010;24(1):73-4.

19. Rasi A, Soltani-Arabshahi R, Shahbazi N. Skin tag as a cutaneous marker for impaired carbohydrate metabolism: a case-control study. International journal of dermatology. 2007;46(11):1155-9.

20. Demir S, Demir Y. Acrochordon and impaired carbohydrate metabolism. Acta diabetologica. 2002;39(2):57-9.

21. Shah R, Jindal A, Patel N. Acrochordons as a cutaneous sign of metabolic syndrome: a case-control study. Ann Med Health Sci Res. 2014;4(2):202-5.

22. Salazar MR, Carbajal HA, Espeche WG, Dulbecco CA, Aizpurua M, Marillet AG, et al. Relationships among insulin resistance, obesity, diagnosis of the metabolic syndrome and cardio-metabolic risk. Diabetes \& vascular disease research. 2011;8(2):109-16.

23. von Elm E, Altman DG, Egger M, Pocock SJ, Gotzsche PC, Vandenbroucke JP. Strengthening the Reporting of Observational Studies in Epidemiology (STROBE) statement: guidelines for reporting observational studies. Bmj. 2007;335(7624):806-8.

24. Friedewald WT, Levy RI, Fredrickson DS. Estimation of the concentration of low-density lipoprotein cholesterol in plasma, without use of the preparative ultracentrifuge. Clinical chemistry. 1972;18(6):499-502.

25. McLaughlin T, Reaven G, Abbasi F, Lamendola C, Saad M, Waters D, et al. Is there a simple way to identify insulin-resistant individuals at increased risk of cardiovascular disease? Am J Cardiol. 2005;96(3):399-404.

26. Haffner SM, Stern MP, Dunn J, Mobley M, Blackwell J, Bergman RN. Diminished insulin sensitivity and increased insulin response in nonobese, nondiabetic Mexican Americans. Metabolism. 1990;39(8):842-7.

27. Finucane FM, Luan J, Wareham NJ, Sharp SJ, O'Rahilly S, Balkau B, et al. Correlation of the leptin:adiponectin ratio with measures of insulin resistance in non-diabetic individuals. Diabetologia. 2009;52(11):2345-9.

\section{Supplementary Files}

This is a list of supplementary files associated with this preprint. Click to download.

- STROBEchecklist181219.docx

- FangCroweSuppTablesS1S3191219.docx 